\title{
High enantioselectivity in the asymmetric hydrogenation of ketones by a supported Pt nanocatalyst on a mesoporous modified MCM-41 support
}

\author{
Susmit Basu* \\ CAT Catalytic Center, RWTH-Aachen University, Worringerweg 2, 52074 Aachen, Germany
}

\section{A R T I C L E I N F O}

Article history:

Received 8 November 2014

Accepted 14 December 2014

Published 20 April 2015

\section{Keywords:}

MCM-41

Platinum nanotube

Functionalized silica

Chiral modifier

Enantioselective hydrogenation

\begin{abstract}
A B S T R A C T
Catalysts containing metal nanotubes were prepared by the adsorption of platinum metal nanotubes onto functionalized and modified silica surfaces (MCM-41 and fumed silica). (3-Chloropropyl)trimethoxysilane and cinchonidine were used for functionalization and modification, respectively. Potassium chloroplatinate was used as the metal precursor to impregnate platinum metal nanotubes on the pretreated functionalized and modified silica surfaces. The solid catalysts were characterized by ESEM, TEM, EDAX, and XPS. The MCM-41 supported platinum nanotube catalyst showed $>98 \%$ to $\sim 100 \%$ enantioselectivity towards the hydrogenation of a range of pharmaceutically important chemicals such as methyl pyruvate, ethyl pyruvate, and acetophenone with nearly full conversion.
\end{abstract}

(C) 2015, Dalian Institute of Chemical Physics, Chinese Academy of Sciences. Published by Elsevier B.V. All rights reserved.

\section{Introduction}

In recent years, material chemistry has flourished in all directions from the macro- to nano-world based on nanotechnology, and it is expected to be more prominent in the coming future. Since the discovery of graphite nanotubes, many one dimensional nanotube materials have attracted attention in the last few decades [1]. These nanotube materials have inherent excellent chemical and physical properties, which can be utilized in chemical engineering and catalysis [2-15]. Efforts have been extended towards the synthesis and characterization of carbon-free nanotubes on a solid support because of their wide applications ranging from industry to biology [16-24]. Elemental metal and metal oxides have been used in the synthesis of nanotubes and nanofibers as they can exert very interesting physicochemical and catalytic properties [25-28].

Silica is an interesting and much studied catalyst support. The reason behind the enormous popularity of the silica sup- port is that it can be modified effectively to produce supports with varying physicochemical properties. During the last two decades, many silica supported catalytic systems using cobalt, ruthenium, rhodium, molybdenum, platinum, palladium, etc., metal precursors have been developed and studied [29-35]. In view of the success with these kinds of materials in catalysis, supported metal catalysts are a hot topic to the modern day catalyst researchers.

The report here is directed towards the synthetic methodology of platinum nanotubes on functionalized and modified silica supports, characterization of the synthesized materials by various electron microscopic methods and their use as enantioselective hydrogenation catalysts for important ketonic functionalities to their corresponding alcohol products. This study investigated the effect of platinum nanotubes on the enantioselective hydrogenation of methyl pyruvate (MPV) and acetophenone (ACP) when it was placed on a chirally modified silica support. The most interesting and striking fact to come

* Corresponding author. Tel: +49 24180 28596; Fax: +49 24180 22593; E-mail: susmitbasu@gmail.com

DOI: 10.1016/S1872-2067(14)60279-5 | http://www.sciencedirect.com/science/journal/18722067 | Chin. J. Catal., Vol. 36, No. 4, April 2015 


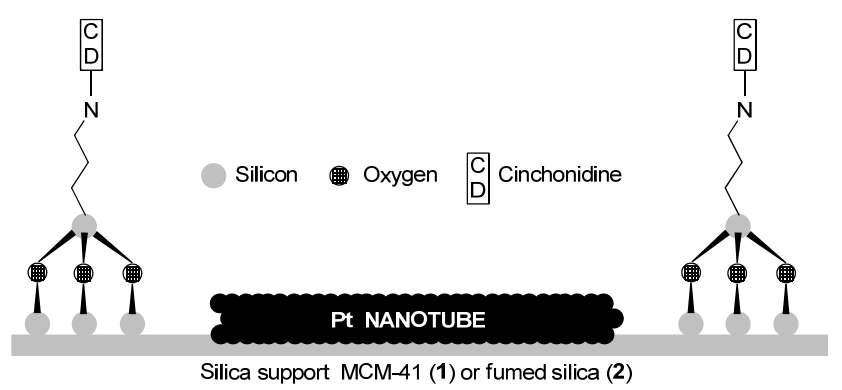

Scheme 1. Schematic representation of the silica supported platinum nanotube containing heterogeneous catalysts.

out from this study was that the platinum nanotube on a functionalized and modified MCM-41 support (1) gave excellent enantioselectivity and conversion, which were higher than obtained with its fumed silica analog (2) (Scheme 1). Experimental results from the hydrogenation reaction revealed that with (2), moderate enantioselectivity ( $50 \%)$ was obtained although the conversion was as high as that of (1) for both substrates. In some typical hydrogenation experiments with (1), enantioselectivities as high as 99.5\% (MPV), 97.8\% (ethyl pyruvale; EPV) and $>98 \%$ (ACP) were obtained. These results are superior to previously reported results with a similar kind of silica supported transition metal containing heterogeneous catalysts [36-44].

\section{Experimental}

\subsection{Materials and methods}

All preparation and manipulation were performed using standard Schlenk techniques under a moisture-free argon atmosphere. Solvents were doubly distilled and then dried over sodium-benzophenone (for toluene) or magnesium turnings-iodine (for methanol) and degassed by dry argon bubbling through the solvent before use. $\mathrm{H}_{2} \mathrm{PtCl}_{6}$ was purchased from Johnson-Matthey (London, UK). MPV, methyl lactate, cinchonidine and colloidal silica were purchased from Fluka (Switzerland). ACP, decane, (3-chloropropyl)trimethoxysilane (3-CPTMS), 1-phenylethanol and fumed silica $(0.014 \mu \mathrm{m})$ were purchased from Aldrich (USA). MCM-41 was synthesized according to a literature reported procedure [45]. All the hydrogenation reactions were carried out in four batch reactor autoclaves (100 mL each) from Parr Instrument Company (USA). Hydrogenation reactions with two different substrates were monitored by gas chromatography with an FID detector (Hewlett Packard HP6890 Series autosampler GC system). Conversion and enantioselectivity were calculated by separating the enantiomeric products and the substrate using a chiral capillary column (Cyclodex-B; J\&W Scientific; length $60 \mathrm{~m}$, inner diameter $0.25 \mathrm{~mm}$, film thickness $0.25 \mu \mathrm{m}$ ). The retention time for each reaction mixture component was obtained initially from reference commercial samples. To get the calibration curve for each component, decane was used as external standard.

\subsection{Functionalization and modification of fumed silica}

Fumed silica (1 g, $16.64 \mathrm{mmol}$ ) was dried and activated under vacuum (1.33 Pa) by keeping at $200{ }^{\circ} \mathrm{C}$ for $24 \mathrm{~h}$. The activated silica was treated with 3-CPTMS (15 mL, $82.27 \mathrm{mmol})$ at $190{ }^{\circ} \mathrm{C}$ in an oil bath for another $96 \mathrm{~h}$ under argon. The resulting solid was filtered and washed thoroughly with dried and degassed toluene under argon. The successful functionalization of fumed silica was evident from its distinct color change from milky white to straw yellow. The solid functionalized product was then dried under vacuum over $\mathrm{P}_{2} \mathrm{O}_{5}$. This dried functionalized product $(1 \mathrm{~g}$ ) was then suspended in a dried and degassed methanol-toluene $(V / V=1: 1)$ mixture solvent. Moisture-free CD (1 g, $3.39 \mathrm{mmol}$ ) was then added to the reaction mixture and refluxed with stirring for $96 \mathrm{~h}$ at $110{ }^{\circ} \mathrm{C}$. It was then filtered and washed thoroughly with dry toluene followed by dry methanol under argon. The obtained solid was dried under vacuum over $\mathrm{P}_{2} \mathrm{O}_{5}$ and kept under desiccation in an argon flushed container.

\subsection{Functionalization and modification of MCM-41}

The procedure was as the same as that for the fumed silica described in Section 2.1. The only difference was that the functionalization step was much longer $(160 \mathrm{~h})$ than with fumed silica.

\subsection{Synthesis of catalysts (1) and (2)}

Powdered functionalized and chirally modified silica (1 g) was dispersed in $300 \mathrm{~g}$ of double distilled water. The silica dispersed solution was cooled at $4{ }^{\circ} \mathrm{C}$ by an ice bath. Subsequently $0.2 \mathrm{~g}(0.5 \mathrm{mmol})$ of $\mathrm{H}_{2} \mathrm{PtCl}_{6}$ and $6 \mathrm{~g}$ of $\mathrm{NH}_{4} \mathrm{OH}$ was injected into the solution with moderate stirring. After $10 \mathrm{~min}, 10 \mathrm{~mL}$ of an ice-cold aqueous solution of sodium borohydride (0.132 g, 3.5 mmol) was added to the silica solution. The mixture was stirred gently for another $2 \mathrm{~h}$. By this method, metallic platinum was reduced by sodium borohydride in the presence of aqueous ammonia solution. To generate smaller metallic platinum particles, the metal reduction was conducted at $4{ }^{\circ} \mathrm{C}$. The temperature of the solution was then increased step by step to $80^{\circ} \mathrm{C}$. Many metal nano particles were formed in this way and they were aggregated together to form a platinum nanotube on the silica surface surrounded by the chiral surface modifier. For both MCM-41 and fumed silica, the same method was employed as described above to get chiral catalysts (1) and (2).

\subsection{Thermal activation of catalysts (1) and (2)}

Catalysts (1) or (2) was placed in a three necked hard glass round-bottomed flask (25 mL) equipped with a Schlenk line. The flask with the solid catalyst was flushed with argon to remove any residual oxygen. The system was evacuated $(0.133$ $\mathrm{Pa}$ ) and then heated at $65-70{ }^{\circ} \mathrm{C}$ for $4 \mathrm{~h}$ under a continuous flow of hydrogen. The catalyst was used in hydrogenation reaction immediately after activation. 


\subsection{Catalytic experiments with activated catalysts (1) and (2)}

All catalytic reactions were carried out at $50{ }^{\circ} \mathrm{C}$ in $25 \mathrm{~mL}$ of methanol solvent in a stainless steel autoclave reactor equipped with a gas inlet and outlet, pressure gauge, mechanical stirrer and temperature controller thermocouple. The system was controlled by computerized software and an electronic motherboard unit. For each reaction, $70 \mathrm{mg}(0.1 \mathrm{mmol}$ of catalytically active Pt metal) of solid catalyst and $100 \mathrm{mmol}$ of substrate were used. The catalytic hydrogenation reaction was carried out under $5 \mathrm{MPa}$ of hydrogen and the reaction time was fixed at $2 \mathrm{~h}$. At the end of the catalytic runs, the reaction mixture was analyzed by GC and the conversion was calculated on the basis of the areas of the starting material and product using calibration curve calculations.

\section{Results and discussion}

Report is there for the use of trialkoxy chloropropylsilane and amines (like ephedrine) for the functionalization and chiral modification of MCM-41, respectively [46]. This material was used as a chiral catalyst in the alkylation of benzaldehyde but only showed moderate enantioselectivity. The structures for catalysts (1) and (2) proposed are shown in Scheme 1. Each step starting from the synthesis of the solid support up to the solid catalysts used after catalysis was studied by different instrumental techniques. MCM-41 and fumed silica surface without additives, and after functionalization and chiral modification were studied by powder XRD, BET surface area porosity, ESEM, EDAX and TEM. In all cases, the silica framework retained was similar to the pure solid support. Most importantly, the powder XRD pattern matched well with the reported characteristic low $2 \theta$ angle diffraction pattern of MCM-41 [47]. The BET surface area and porosity measurement for (1) and (2) also matched well with the previously reported values [48]. For (1), 90\% of the pore volume was in the range of 1 to $2 \mathrm{~nm}$, while $65 \%$ of the pore volume for (2) fell within 2 to $8 \mathrm{~nm}$. This suggested a more regular pore distribution of (1) than (2). Moreover, the TEM analysis (Fig. 1(a)) showed the basic characteristics of MCM-41 fringes visible on the surface. The TEM analysis of (1) and (2) showed the formation of the platinum metal nanotubes on the surface of the solid support. The nanotubes have the average thickness of 8 to $10 \mathrm{~nm}$ (Fig. 1(b) and (c)). The spot EDAX analysis of these tube gave $\sim 100 \%$ counts for platinum metal, which confirmed the purity of the metal nanotube formed on the surface (Fig. 1(c)).

From the chemical analysis, it was found that the extent of functionalization (3.0 mmol/g, surface EDAX analysis) and chiral modification (2.8 mmol/g, from CHNS/O analysis) and platinum metal $(0.48 \mathrm{mmol} / \mathrm{g}$, from ICP-AES and surface EDAX analysis) load were nearly the same for both (1) and (2). The extent of functionalization and chiral modification revealed that most of the chloropropyl groups were capped with a chiral modifier like CD.

The XPS analysis for (1) and (2) showed the presence of one major species of platinum (with binding energy $71 \mathrm{eV}$ ) on the surface, which matched well with the reported binding energy value for platinum crystallites [49]. After deconvolution, a smaller band was observed in the XPS spectrum with a higher binding energy (72 eV). This species was possibly due to the formation of $\mathrm{PtCl}_{2}\left(\mathrm{Pt}^{2+}\right)$ on the catalyst surface which has surface chloride.

The catalytic experiments were carried out with MPV, EPV and ACP substrates. In all cases, the hydrogenation reaction was almost complete after 2 to $3 \mathrm{~h}$. For all the substrates, the enantioselectivity reached the highest point after 1 to $1.5 \mathrm{~h}$ and this remained the same up to complete conversion. The most interesting fact from these catalytic experiments was that (2) showed some enantioselectivity (50\% on average) in the hydrogenation of MPV and ACP. There has been reported that with platinum cluster based catalysts, there was no observable enantioselectivity [29]. The reason behind the higher enantioselectivity of (2) was because of the accumulation of more chirally modified site on the surface with the active platinum metal nano-tubular catalytic site. This was also why (1) showed much higher conversion and activity (TOF values) with more excellent enantioselectivity than (2), and the reported platinum cluster-based catalyst mentioned earlier. This revealed the importance of an optimized solid support in heterogeneous chiral catalysis. The unique pore arrangement on the mesoporous MCM-41 surface made the support more effective for binding of the chiral modifier entity in its one spatial orienta-

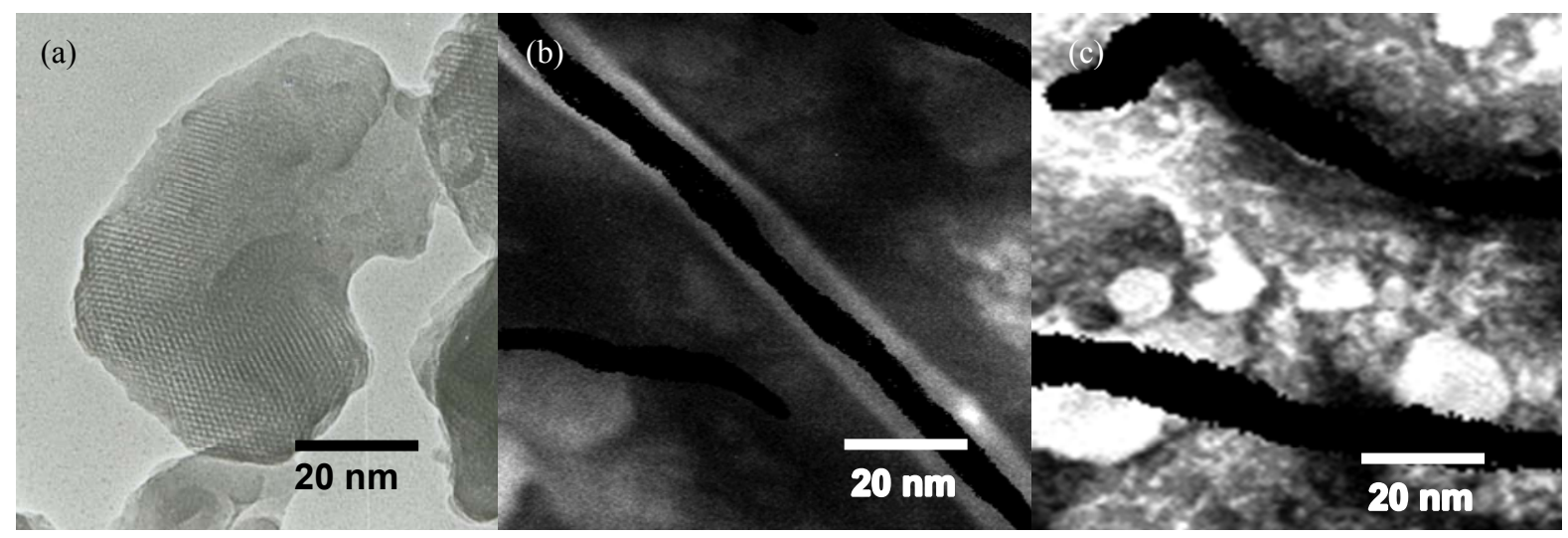

Fig. 1. TEM images of (a) MCM-41 surface with visible surface fringes, (b) surface of catalyst (1) containing a Pt nanotube and (c) surface of catalyst (2) containing a Pt nanotube. 
Table 1

Catalytic results over catalysts (1) and (2).

\begin{tabular}{|c|c|c|c|c|}
\hline Substrate & Catalyst & Conversion (\%) & $e e(R$ isomer $)(\%)$ & TOF $\left(\mathrm{h}^{-1}\right)$ \\
\hline $\mathrm{OMe}$ & (1) & 100 & 99.2 & 666 \\
\hline $\begin{array}{r}\mathrm{O} \\
(\mathrm{MPV})\end{array}$ & (2) & 90 & 53 & 256 \\
\hline & (1) & 100 & 98 & 556 \\
\hline (ACP) & (2) & 96 & 49 & 285 \\
\hline & (1) & 100 & 99.8 & 575 \\
\hline $\begin{array}{r}0 \\
\text { (EPV) } \\
\end{array}$ & (2) & 88 & 58 & 220 \\
\hline
\end{tabular}

tion than was the case with fumed silica (which has a much more disordered pore distribution). Table 1 summarizes the observed catalytic results with MPV, EPV and ACP in presence of (1) and (2).

Catalysts (1) and (2) were studied for their recyclability. During this study, each time after the catalytic hydrogenation reaction, the catalyst was recovered by simple filtration and washed with dry methanol. The washed and recovered catalyst was then dried overnight under vacuum at $100{ }^{\circ} \mathrm{C}$. The recycling experiment was performed under identical hydrogenation reactions as before. The results showed a decrease in the conversion (in the same reaction time as the first cycle) and enantioselectivity from the first to second to third catalytic cycle for both catalysts (1) and (2) in the presence of MPV and ACP substrates. The decrease in enantioselectivity was more striking than the decrease in conversion. The well-known leaching behavior of these kinds of catalyst was most probably the reason behind the decreased catalytic activity in the recycling experiments. The results of the recycling experiments are shown in Table 2 .

\section{Conclusions}

This report showed that treating of the silica support with alkoxy-chlorosilane functionalizer and chiral amine modifier made it act as a chiral solid support. The deposition of platinum metal on the chirally modified silica support in the form of a nanotube morphology produced a unique chiral heterogeneous catalyst. Excellent enantioselectivity with very high conversion and catalytic activity was obtained with (1). A similarly treated fumed silica based catalyst (2) showed moderately high enantioselectivity with very high conversions. Experiments on catalyst recycle (three times) showed the influence of leaching as a decrease in conversion and enantioselectivity for catalysts (1) and (2).

\section{Acknowledgments}

The research infrastructure and financial supports from the RWTH-Aachen University are highly acknowledged.

\section{References}

[1] Iijima S. Nature, 1991, 354: 56

[2] Che G L, Lakshmi B B, Martin C R, Fisher E R. Langmuir, 1999, 15: 750

[3] Li Y D, Chen J L, Ma Y M, Zhao J B, Qin Y N, Chang L. Chem Commun, 1999: 1141

[4] Zhang Y, Zhang H B, Lin G D, Chen P, Yuan Y Z, Tsai K R. Appl Catal A, 1999, 187: 213

[5] De Jong K P, Geus J W. Catal Rev-Sci Eng, 2000, 42: 481

[6] Joo S H, Choi S J, Oh I, Kwak J, Liu Z, Terasaki O, Ryoo R. Nature, 2001, 412: 169

[7] Oshima Y, Koizumi H, Mouri K, Hirayama H, Takayanagi K, Kondo Y. Phys Rev B, 2002, 65: 121401

[8] Pawelec B, La Parola V, Navarro R M, Murcia-Mascaros S, Fierro J L G. Carbon, 2006, 44: 84

[9] Corma A, Garcia H. Top Catal, 2008, 48: 8

[10] Baglio V, Di Blasi A, D'Urso C, Antonucci V, Arico A S, Ornelas R, Morales-Acosta D, Ledesma-Garcia J, Godinez L A, Arriaga L G, Alvarez-Contreras L. J Electrochem Soc, 2008, 155: B829

[11] Tu W W, Lei J P, Ju H X. Electrochem Commun, 2008, 10: 766

[12] Nath D, Banerjee P. Environ Toxicol Pharmacol, 2013, 36: 997

[13] Jiao Q Z, Hao L, Shao Q Y, Zhao Y. Carbon, 2013, 61: 647

[14] Mubarak N M, Abdullah E C, Jayakumar N S, Sahu J N. J Ind Eng Chem, 2014, 20: 1186

[15] Conley M P, Coperet C, Thieuleux C. ACS Catal, 2014, 4: 1458

[16] Zhou H J, Zou F M, Koh K, Lee J. J Biomed Nanotechnol, 2014, 10: 2921

[17] Tang Q, Zhang Q E, Jiang Y, Li J S, Zheng J, Li Y H, Yang R H, Tan W H. ACS Appl Mater Interf, 2014, 6: 13470

[18] Bousslama W, Sieber B, Elhouichet H, Gelloz B, Addad A, Ferid M. J Phys D, 2013, 46: 505104

[19] Von Bargen C D, MacDermaid C M, Lee O S, Deria P, Therien M J, Saven J G. J Phys Chem B, 2013, 117: 12953

[20] Miller J. Phys Today, 2012, 65(6): 22

[21] Lee S H, Sung J H, Park T H. Ann Biomed Eng, 2012, 40: 1384

[22] Panthani M G, Korgel B A. Annu Rev Chem Biomol Eng, 2012, 3: 287

[23] Kameta N, Masuda M, Shimizu T. Kobunshi Ronbunshu, 2010, 67: 560

[24] Chakoli A N, Wan J, Feng J T, Amirian M, Sui J H, Cai W. Appl Surf Sci, 2009, 256: 170

[25] Hochbaum A I, Fan R, He R R, Yang P D. Nano Lett, 2005, 5: 457

[26] Shin J C, Kim D Y, Lee A, Kim H J, Kim J H, Choi W J, Kim H S, Choi K

Table 2

Catalytic results over catalysts (1) and (2) in the catalyst recycling experiments.

\begin{tabular}{|c|c|c|c|c|c|c|c|}
\hline \multirow{2}{*}{ Substrate } & \multirow{2}{*}{ Catalyst } & \multicolumn{2}{|c|}{ 1st Cycle } & \multicolumn{2}{|c|}{ 2nd Cycle } & \multicolumn{2}{|c|}{ 3rd Cycle } \\
\hline & & Conversion (\%) & $e e^{a}(\%)$ & Conversion (\%) & $e e(\%)$ & Conversion (\%) & $e e(\%)$ \\
\hline \multirow{2}{*}{ MPV } & (1) & 100 & 99.2 & 88 & 67.8 & 56 & 45.6 \\
\hline & (2) & 90 & 53 & 77 & 21.5 & 46.8 & 12.3 \\
\hline \multirow{2}{*}{ ACP } & (1) & 100 & 98 & 83 & 59.4 & 39.4 & $<1$ \\
\hline & $(2)$ & 96 & 49 & 80 & 18 & 38 & 5 \\
\hline
\end{tabular}

${ }^{a} R$-isomer is the major isomer. 


\section{Graphical Abstract}

Chin. J. Catal., 2015, 36: 634-638 doi: 10.1016/S1872-2067(14)60279-5

\section{High enantioselectivity in the asymmetric hydrogenation of ketones by a supported Pt nanocatalyst on a mesoporous modified MCM-41 support}

Susmit Basu*

RWTH-Aachen University, Germany

An MCM- 41 based Pt nanocatalyst gave the best results with $>99 \%$ and $98 \%$ enantioselectivity in the asymmetric hydrogenation of methyl pyruvate and acetophenone, respectively.

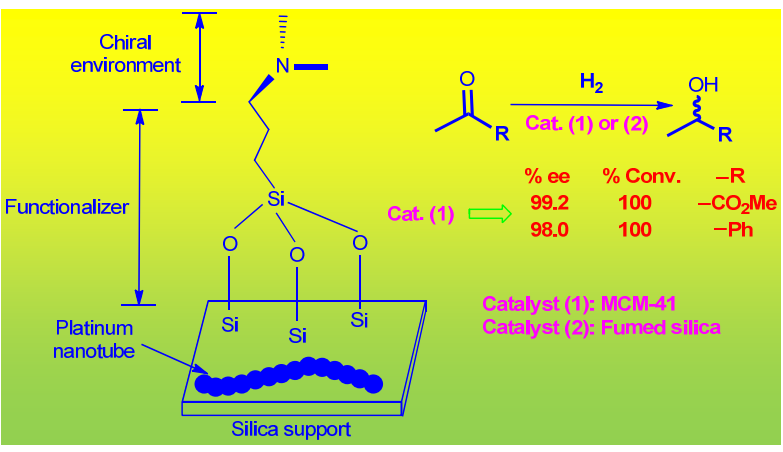

ChemCatChem, 2011, 3: 741

[39] Indra A, Doble M, Bhaduri S, Lahiri G K. ACS Catal, 2011, 1: 511

[40] Török B, Balázsik K, Török M, Szöllösi G, Bartók M. Ultrason Sonochem, 2000, 7: 151

[41] Bartók M, Balázsik K, Szöllösi G, Bartók T. J Catal, 2002, 205: 168

[42] Schmidt E, Vargas A, Mallat T, Baiker A. J Am Chem Soc, 2009, 131: 12358

[43] Schmidt E, Hoxha F, Mallat T, Baiker A.J Catal, 2010, 274: 117

[44] Hoxha F, Schimmoeller B, Cakl Z, Urakawa A, Mallat T, Pratsinis S E, Baiker A. J Catal, 2010, 271: 115

[45] Beck J S, Vartuli J C, Roth W J, Leonowicz M E, Kresge C T, Schmitt K D, Chu C T W, Olson D H, Sheppard E W, McCullen S B, Higgins J B, Schlenker J L. J Am Chem Soc, 1992, 114: 10834

[46] Jyothi T M, Kaliya M L, Herskowitz M, Landau M V. Chem Commun, 2001: 992

[47] Mokaya R. Chem Commun, 2001: 1092

[48] Basu S, Paul H, Gopinath C S, Bhaduri S, Lahiri G K. J Catal, 2005, 229: 298

[49] Apai G, Lee S T, Mason M G, Gerenser L J, Gardner S A. J Am Chem Soc, 1979, 101: 6880

[37] Crosman A, Hölderich W F. J Catal, 2005, 232: 43

[38] Böttcher S, Hoffmann C, Räuchle K, Reschetilowski W. 